\title{
Potentially Resectable mCRC-treated with Cetuximab Combined with Chemotherapy
}

\author{
Ying Kong, Liang Hong and Xiaocheng Xu \\ Department of Oncology, The First People's Hospital of Xiaoshan District, Hangzhou, China
}

\begin{abstract}
Surgical resection of a potentially resectable metastatic colorectal cancer ( $\mathrm{MCRC}$ ) may lead to additional clinical benefits for the patients. In some cases, patients with initially unresectable lesions can be converted to resectable ones after induction of chemotherapy; and these patients are primarily treated with fluorouracil-based systemic chemotherapy preoperatively. However, the optimal protocol for neoadjuvant therapy has not been determined yet, and it remains a source of controversy about whether systemic chemotherapy combined with cetuximab can increase the surgical resection rate and obtain more clinical benefits. Metastatic CRC patients that received chemotherapy combined with cetuximab were compared with those undergoing chemotherapy alone. The hazard ratio (HR) and odds ratio (OR) were used as the efficacy indicators. The $95 \%$ confidence interval $(95 \% \mathrm{Cl})$ was used as interval estimation method. Seven studies including 1,895 patients were selected. Compared with patients undergoing chemotherapy, the patients receiving systemic chemotherapy combined with cetuximab of the R0 resection was not improved $(O R=1.25 ; 95 \% \mathrm{Cl}, 0.76-2.06 ; p=0.08)$; The progression-free survival (PFS) of the patients received chemotherapy combined with cetuximab was slightly longer than those received chemotherapy alone $(\mathrm{HR}=0.88$; $95 \% \mathrm{Cl}$, $0.77-1 ; p=0.005)$; and the overall survival (OS) of patients undergoing systemic chemotherapy combined with cetuximab was not prolonged relative to that in patients receiving chemotherapy alone $(\mathrm{HR}=0.98 ; 95 \% \mathrm{Cl}, 0.86-1.11 ; \mathrm{p}=0.04)$. Compared with patients receiving chemotherapy alone, the surgical resection rate and PFS are not increased in patients undergoing chemotherapy combined with cetuximab, but the OS is slightly prolonged.
\end{abstract}

Key Words: Chemotherapy, Cetuximab, Colorectal cancer, Metastatic tumor, Surgery.

How to cite this article: Kong Y, Hong L, Xu X. Potentially Resectable mCRC-treated with Cetuximab Combined with Chemotherapy. J Coll Physicians Surg Pak 2020; 30(11):1206-1212.

\section{INTRODUCTION}

Most patients with mCRC cannot be cured, but the metastatic lesion is limited to the liver and/or lung, and local recurrence or the localised intraperitoneal lesions of $\mathrm{mCRC}$ can be cured surgically. Dramatic progresses have been attained in $\mathrm{MCRC}$ over the past 15 years, which can be evidenced by the remarkably improved outcomes for MCRC patients, thanks to the increased surgical opportunities for the potentially resectable patients and the more effective chemotherapy. ${ }^{1}$ For $\mathrm{mCRC}$ restricted to the liver, the 5 -year survival after resection is $24-58 \%$, with the average of $40 \%{ }^{2,3}$ Unfortunately, only $\leq 20 \%$ patients with liver metastasis alone can undergo potentially radical resection, while most patients are not the candidates of surgery due to tumor size and site, multifocal nature as well as otherfactors.

Correspondence to: Dr. Ying Kong, Department of Oncology, The First People's Hospital of Xiaoshan District, Hangzhou, China

E-mail: kongying750@163.com

Received: December 14, 2019; Revised: May 29, 2020;

Accepted: May 29, 2020

DOI: https://doi.org/10.29271/jcpsp.2020.11.1206
Notably, some patients with initially unresectable lesions can be converted to resectable ones after induction of chemotherapy in recent years. Among patients with initially unresectable liver metastases, $12-33 \%$ have achieved sufficient objective remission that allows for subsequent complete (R0) resection, with the 5-year survival of 30-35\%, which is far superior to that of chemotherapy alone, as reported in literature. ${ }^{4}$

Epidermal growth factor receptor (EGFR) has been established to be the target of anti-tumor agents, ${ }^{5}$ while cetuximab is a monoclonal immunoglobulin $\mathrm{Gl}$ antibody against EGFR, which can improve the PFS and OS when applied alone or combined with irinotecan in the third-line treatment for $\mathrm{MCRC}$. $^{6-8}$ Typically, high surgical resection rate is reported in FOILFOXIRI among patients with an initial unresectable liver metastasis. According to literature for the wild-type KRAS of mCRC, the addition of cetuximab into the oxaliplatin- or irinotecan-based chemotherapy can increase the number of potentially resectable patients, in the meantime of improving PFS and OS. ${ }^{9-11}$ However, the opposite is also true. A EPOC trial study (FOLFOX combined with or without cetuximab) shows that the PFS for patients with liver metastases, which are potentially resectable, is markedly poor after FOLFOX combined with cetuximab treatment (14.8 vs. 24.2 months). ${ }^{12}$ 
Table I: Characteristics of studies included in meta-analysis.

\begin{tabular}{|c|c|c|c|c|c|}
\hline First author & Patients (n) & Arm A & Arm B & Comparative outcomes & Design \\
\hline Cutsem $2009^{10}$ & 348 & FOLFIRI + cetuximeb & FOLFIRI & PFS, OS, R0 resection & RCT \\
\hline Bokemeyer $2009^{9}$ & 134 & FOLFOX4 + cetuximeb & FOLFOX4 & PFS, R0 resection & RCT \\
\hline Timothy S $2011^{15}$ & 715 & XELOX + cetuximeb & XELOX & OS, RO resection & RCT \\
\hline Magne $2012^{14}$ & 194 & FLOX + cetuximab & FLOX & PFS, OS & RCT \\
\hline Ye $2013^{11}$ & 138 & FOLFIRI + cetuximab & FOLFIRI & PFS, OS, R0 resection & RCT \\
\hline Primrose $2014^{12}$ & 257 & chemotherapy + cetuximab & chemotherapy & PFS,OS & RCT \\
\hline Sia^n A $2016^{16}$ & 109 & chemotherapy + cetuximab & chemotherapy & R0 resection & RCT \\
\hline
\end{tabular}

\begin{tabular}{|c|c|c|c|c|c|c|c|c|c|}
\hline \multirow[t]{2}{*}{ nn } & \multicolumn{3}{|c|}{$\mathrm{RO}$ resection } & \multicolumn{3}{|c|}{ PFS } & \multicolumn{3}{|c|}{ OS } \\
\hline & OR & $95 \% \mathrm{Cl}$ & p-value & HR & $95 \% \mathrm{Cl}$ & p-value & HR & $95 \% \mathrm{Cl}$ & p-value \\
\hline Cutsem $2009^{10}$ & 2.81 & $0.73,10.78$ & 0.2004 & 0.68 & $0.5,0.94$ & 0.048 & 0.84 & $0.64,1.11$ & 0.03 \\
\hline Bokemeyer $2009^{9}$ & 0.39 & $0.09,1.64$ & 0.1315 & 0.93 & $0.70,1.23$ & 0.163 & - & - & - \\
\hline Timothy S $2011^{15}$ & 0.86 & $0.37,2.02$ & 0.7363 & - & - & - & 1.04 & $0.87,1.23$ & 0.67 \\
\hline Magne $2012^{14}$ & 0.92 & $0.41,2.07$ & 0.8357 & 0.89 & $0.72,1.1$ & 0.66 & 1.14 & $0.80,1.61$ & 0.48 \\
\hline Ye $2013^{11}$ & 4.36 & $1.52,12.55$ & 0.0063 & 0.6 & $0.41,0.87$ & 0.004 & 0.54 & $0.33,0.89$ & 0013 \\
\hline Primrose $2014^{12}$ & 0.93 & $0.45,1.89$ & 0.8363 & 1.48 & $1.04,2.12$ & 0.03 & 1.49 & $0.86,2.6$ & 0.16 \\
\hline Sia^n A $2016^{16}$ & 1.53 & $0.65,3.58$ & 0.3318 & - & - & - & - & - & - \\
\hline
\end{tabular}

This study is aimed to analyse the potentially resectable $\mathrm{mCRC}$ patients that received cetuximab combined with neoadjuvant chemotherapy (NACT) based on the carefully screened experimental data.

\section{METHODOLOGY}

\section{Search Strategy:}

Electronic databases, including PubMed, Embase, Cochrane, and Web of Science, were systemically retrieved to identify the eligible clinical studies published in English between January 1979 and May 2019, based on the preferred reporting items of the system review and meta-analysis (PRISMA) statement. ${ }^{13}$ In the meta-analysis, the keywords of electronic data literature retrieval include chemotherapy, "cetuximab", "CRC", "meta-static tumor", and "surgery".

All studies were screened and retrieved by two reviewers ( $Y$ Kong and L Hong) independently. Subsequently, in order to avoid missing any relevant publications, all the original studies, review, and references of relevant articles were listed. Additionally, the references in this article were also retrieved manually, and any difference between them were resolved by discussion.

The study inclusion criteria were as follows, (I) RCTs were selected in which patients were assigned to two groups (namely, the cetuximab combined with chemotherapy group, and the chemotherapy group). (II) Studies in which patients were pathologically confirmed as the wild type CRC KRAS; and those with available data on the R0 removal rate and at least one of PFS and OS, were also selected. The study exclusion criteria were non-RCTs; duplicate publications; and (III) studies with available data on the R0 removal rate and at least one of the following items: PFS and OS. The study exclusion criteria were shown below: (I) non-RCTs; (II) duplicate publications; and (III) studies that enrolled patients aged less than 18 years.

\section{Data Extraction:}

The following information including year of publication, number of patients, chemotherapy regimen, R0 resection, PFS and OS, which were extracted from each article.

All the selected articles were reviewed by $Y$ Kong and $L$ Hong. In addition, the study quality was evaluated according to the Tang's and Black's methods, which included 27 criteria to evaluate the RCTs and non-RCTs.

\section{Statistical Analysis:}

Meta-analysis was performed using Review Manager 5.3 (The Nordic Cochrane Centre, The Cochrane Collaboration, Copenhagen, 2014). To statistically evaluate the surgical resection rate of cetuximab combined with chemotherapy, the ORs of the number of surgical patients in experimental group and control group, together with the relevant standard errors, were extracted from the enrolled studies providing these statistical data. Moreover, the HRs and related standard errors for PFS and/or OS were also extracted from the articles that include these statistics, so as to statistically assess the prognostic effect of cetuximab plus chemotherapy. Results with $p<0.05$ were defined as statistically significant. In addition, the statistics heterogeneity was assessed by Chi-square test and quantified by 12 statistics. In the absence of heterogeneity, the fixed effect model was adopted; otherwise, the random effect model was adopted $\left(p<0.10,1^{2}>50 \%\right.$ ). 


\section{RESULTS}

According to the search strategy, 69 articles were found, with a total of 1,895 patients. Seven RCTs studies were included in this meta-analysis (Figure 1). In 2009, Van Cutsem et al. ${ }^{10}$ had randomly assigned patients with unresectable EGFR-positive $\mathrm{MCRC}$ to receive FOLFIRI (fluorouracil, leucovorin, and irinotecan) alone or FOLFIRI combined with cetuximab. Among them, 599 were treated with cetuximab combined with FOLFIRI, while the remaining 599 had received FOLFIRI alone. The median PFS was 8.0 and 8.9 months in the cetuximab-FOLFIRI and FOLFIRI groups, respectively. In addition, the HR of PFS between cetuximab-FOLFIRI and FOLFIRI groups was 0.68(95\% Cl 0.50-0.94. $\mathrm{p}=0.048)$. Besides, the median OS was 19.9 and 18.8 months respectively in the cetuximab-FOLFIRI group and FOLFIRI group, there are no statistical significance in the OS rate between these two groups $(\mathrm{HR}=0.84 ; 95 \% \mathrm{Cl} 0.64-1.11 ; \mathrm{p}=0.03)$. In addition, the surgical rate of metastatic tumor in cetuximab-FOLFIRI group was higher than another group $(7.0 \% \mathrm{vs}$. $3.7 \%)$, and the $\mathrm{R0}$ resection rate before disease progression $(4.8 \%$ vs. $1.7 \%)$ was also higher $(7.0 \%$ vs. $3.7 \%)$. The OR between cetuximab-FOLFIRI and FOLFIRI groups was 0.39, $95 \% \mathrm{Cl}, 0.09-1.64 ; p=0.131$ (Table II).

In 2009, Bokemeyer et al. had evaluated whether the combination of cetuximab with oxaliplatin, folic acid calcium and fluorouracil (FOLFOX-4) as the first-line treatment for mCRC could attain superior overall response rate (ORR) to that of FOLFOX-4 alone. ${ }^{9}$ Their results suggested that, for patients with wild-type KRAS, the combination of cetuximab with FOLFOX-4 could dramatically increase the effective rate and reduce the risk of disease progression, relative to that of FOLFOX-4 alone. No significant difference in PFS was found between these two groups for secondary endpoints. Moreover, the R0 resection rate was over 2-fold higher in wild-type KRAS tumors of the patients that received cetuximab combined with FOLFOX-4 than that in those treated with FOLFOX-4 alone.

Another RCT published in 2013 was about the KRAS wild-type unresectable synchronous liver metastasis in CRC patients. ${ }^{11} \mathrm{~A}$ total of 138 cases were enrolled in that study; among them, 70 patients received chemotherapy FOLFIRI or mFOLFOX6 + cetuximab, while the remaining 68 cases were randomly assigned to chemotherapy alone group. All patients were followed up for an average of 25.0 months, with the 3-year OS rate of 30\%, and the median survival time (MST) of 24.4 months. The OS rate of patients in chemotherapy group was higher than that in combination of cetuximab group (3-year survival rate: $41 \%$ vs $18 \%$ ); meanwhile, the MST was 30.9 vs. 21.0 months $(H R=0.54 ; p=$ $0.013)$, and the median PFS was 10.2 vs 5.8 months $(H R=0.60 ; p=0.04)$. Besides, the $R 0$ resection rate of liver metastasis was $25.7 \%$ ( $18 / 70$ cases) in group $A$ and $7.4 \%$ $(5 / 68$ cases) in group $B(p<0.01)$, respectively. Moreover, neither the mFOLFOX6 nor the FOLFIRI regimen could result in significant survival benefits for patients in subgroup A (Table II).

In the 2011 (MRC) COIN trial, ${ }^{14}$ a total of 1630 advanced CRC patients naive to chemotherapy were randomly assigned to oxaliplatin and fluorouracil group and the combined cetuximab group. Among them, 367 patients in oxaliplatin and fluorouracil group had wild-type KRAS tumors; while such figure was 362 in another group, and no statistical difference was found in OS between the two groups (the median survival was 17.9 months in control group and 17.0 months in cetuximab group). Similarly, no statistical difference was found in PFS (8.6 months in control group, and 8.6 months in cetuximab group). In subgroup analysis, PFS was improved in patients with no or only one metastatic lesion $(H R=0.73$, 0.55-0.97, $p=0.036$ ), but it was not enhanced in patients with at least two metastases $(H R=1.07,0.86-1.33$, $p=0.036$ ). With regard to the surgical analysis of metastatic tumors, the resection rates in patients with wild-type KRAS were $13 \%$ at baseline $(n=12 / 91)$ and $15 \%$ in control group $(n=13 / 87)$, and the potential liver resection rate was not increased (Table II).

In the 2012 NORDIC-VII study, ${ }^{15}$ altogether 571 patients were enrolled and randomised as FLOX alone or cetuximab plus FLOX group. In patients with wild-type KRAS tumors, the survival curves of these two groups were similar, with the median PFS of 8.7 months. In addition, the median OS of these two groups was 22.0 months, and difference in the survival curve between them was not statistically significant. The overall $\mathrm{R} 0$ resection rate of metastatic tumors was $8 \%$, among which, the R0 resection rate in FLOX group was $8 \%$, while that in cetuximab plus FLOX group was 11\%, and the difference between these two groups was not statistically significant (Table II).

Additionally, a new EPOC RCT in 2014 had evaluated the effect of standard chemotherapy combined with cetuximab on the resectable KRAS exon type 2 wild-type CRC liver metastasis (CRCLM). ${ }^{12,16}$ Among them, 128 patients were randomised to receive chemotherapy, 129 were treated with chemotherapy plus cetuximab, with the average follow-up of 20.7 months. Their results suggested that the PFS time in chemotherapy combined with cetuximab group was dramatically shorter than that in chemotherapy group alone. Besides, the median OS was 39.1 months in chemotherapy combined with cetuximab group, but not in chemotherapy alone group. In addition, a majority of patients in these two groups had undergone resection, and the difference was not statistically significant (Table II).

Patient characteristics are displayed in Table I. Obviously, the $\mathrm{R} 0$ resection rates reported in these seven studies were not increased in patients undergoing systemic chemotherapy combined with cetuximab relative to those in patients treated with chemotherapy alone $(O R=1.25 ; 95 \%$ $\mathrm{Cl}, 0.76-2.06 ; \mathrm{p}=0.08$ ), and statistically moderate hetero- 
geneity was detected $\left(I^{2}=48 \%\right)$. In subgroup analysis, the $\mathrm{R} 0$ resection rates in studies involving the initially resectable metastatic tumors were not increased $(\mathrm{OR}=0.95 ; 95 \% \mathrm{Cl}$, 0.65 -1.39; $p=0.61, I^{2}=0 \%$ ). For patients with initially unresectable subgroup of metastatic tumors, the $\mathrm{R} 0$ resection rate in systemic chemotherapy combined with cetuximab group was higher than that in chemotherapy group (OR= 3.69; $95 \% \mathrm{Cl}, 1.61-8.47 ; p=0.62, \mathrm{l}^{2}=0 \%$, Figure 2).

Moreover, data extracted from five studies suggested that the PFS in systemic chemotherapy combined with cetuximab group was slightly prolonged $(\mathrm{HR}=0.88 ; 95 \% \mathrm{Cl}, 0.77-1.00$; $p=0.05$ ). Additionally, subgroup analysis of the five studies was carried out due to the presence of overall statistical heterogeneity $\left(I^{2}=73 \%\right)$. In subgroup analysis, statistical difference was only observed in cetuximab plus FOFIRI group compared with that in control group $(\mathrm{HR}=0.65 ; 95 \% \mathrm{Cl}$, $0.51-0.82 ; p<0.001$ ); while the results in cetuximab plus FOLFOX group and FOLFOX group were as follows: $(\mathrm{HR}=0.90 ; 95 \% \mathrm{Cl}, 0.76-1.07 ; p=0.25)$. At the same time, the random-effect model was adopted due to the great heterogeneity in the three subgroups $\left(I^{2}=73 \%\right.$, Figure 3$)$.

In the five included studies, compared with chemotherapy treatment alone, the OS in cetuximab plus chemotherapy group was not increased $(\mathrm{HR}=0.88 ; 95 \% \mathrm{Cl}, 0.77-1.00$; $\mathrm{p}=0.005$ (Figure 4). In subgroup analysis, the OS between the two groups of studies recruiting the initially resectable metastatic tumors was not statistically significant different. $\left(\mathrm{HR}=1.09 ; 95 \% \mathrm{Cl}, 0.93-1.27 ; \mathrm{p}=0.46, \mathrm{I}^{2}=0 \%\right)$. Similary, in the initially unresectable subgroup of metastatic tumors of the two groups, there was no statistically significant difference. $\left(\mathrm{HR}=0.76 ; 95 \% \mathrm{Cl}, 0.60-0.96 ; p=0.12\left(I^{2}=58 \%\right)\right.$.

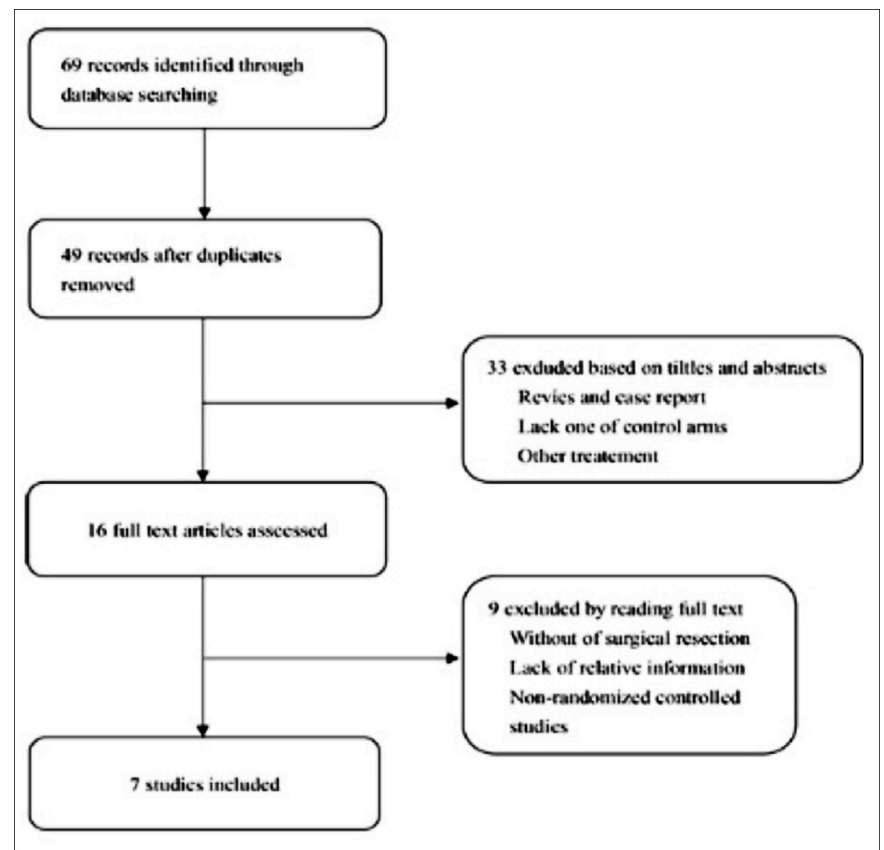

Figure 1: Flow chart of literature selection.

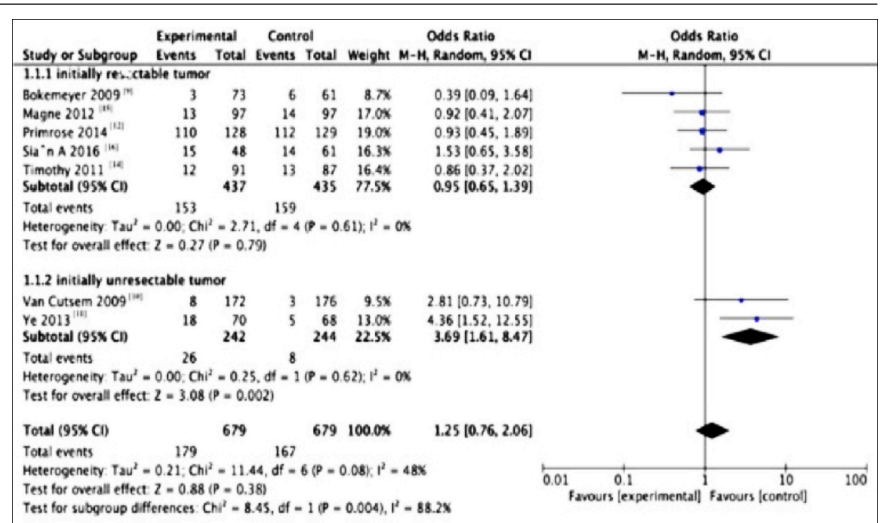

Figure 2: Forest plots for the resuts of meta-analysis for RO resection of the two subgroups.

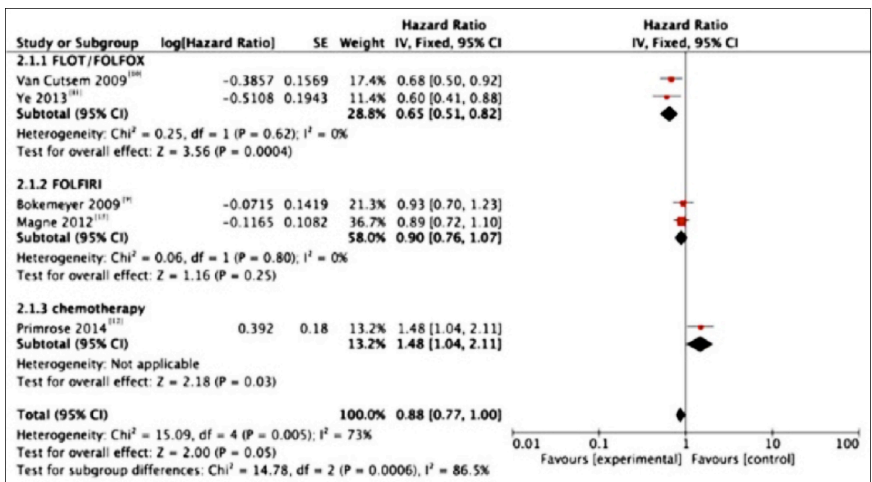

Figure 3: Forest plots for the results of meta-analysis for progression-free surrival (PFS) of the three subgroups.

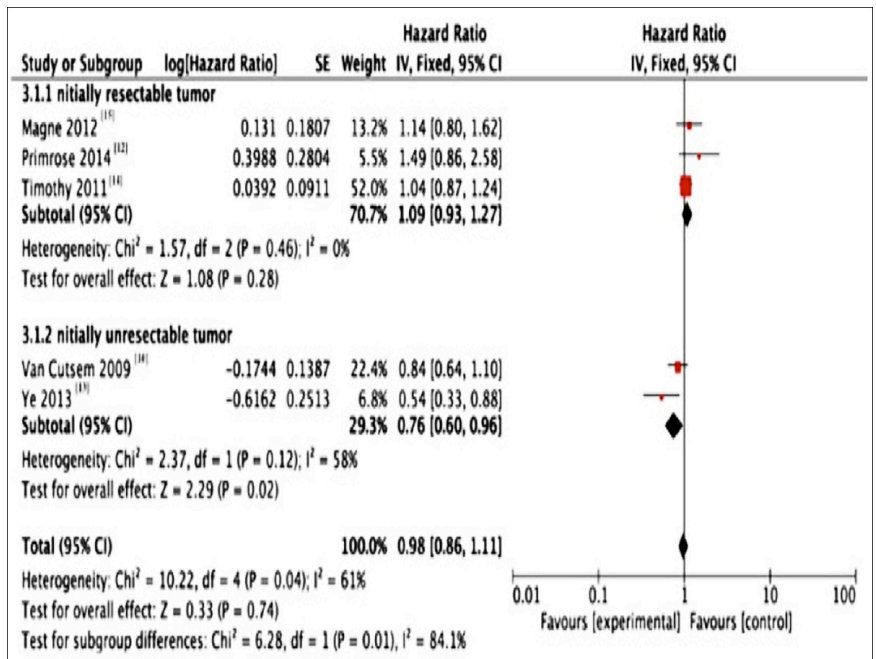

Figure 4: Forest plots for the results of meta-analysis for overall survival (OS) of the two groups.

\section{DISCUSSION}

The aim of this meta-analysis was to evaluate the clinical effect of adding cetuximab to the chemotherapy regimens on patients with potentially resectable CRCLM. In this study, patients with potentially resectable CRCLM receiving cetuximab combined with chemotherapy were compared with those undergoing chemotherapy alone. Prior to the MRC COIN trial, existing evidence had consistently indicated that cetuximab could remarkably improve the PFS, OS and surgical 
resection rate in wild-type KRAS of the patients. Nonetheless, the MRC COIN trial discovered that cetuximab combined with standard chemotherapy would not increase the clinical benefits. ${ }^{14}$ Jones et al. had published a systematic review based on 15 RCTs and cohort studies in $2013,{ }^{17}$ which had examined the role of cetuximab as the first-line therapy for CRCLM. They did not support the routine use of cetuximab for CRCLM upon comprehensive analysis of related literature, which was basically consistent with our results. In the 2010 CELIM study, ${ }^{18}$ the total effective rates of cetuximab plus FOLFIRI and FOLFOX-6 were $68 \%$ and $59 \%$, respectively, and no statistical significance was observed; meanwhile, the R0 removal rates were $38 \%$ and $30 \%$, respectively. In this study, cetuximab combined with chemotherapy was more effective, which could increase the resectability compared with that in historical controls. However, the clinical efficacy of cetuximab could not be evaluated due to the lack of control group or long-term outcome data in this study. Similarly, in the 2010 POCHER trial, ${ }^{19}$ the effects of cetuximab combined with irinotecan, 5-fu, FA and oxaliplatin in NACT on CRCLM were examined. Their results showed that the ORR was $79.1 \%$, the R0 resection rate was $60 \%$, and the overall PFS period was 14 months. Nonetheless, that study had a small sample size without a control group; as a result, the clinical efficacy of cetuximab could not be evaluated. But their study suggested that the combination of cetuximab with chemotherapy might serve as an approach to reduce the disease stage.

In the seven studies included in this metastasis, the $\mathrm{R} 0$ resection rates between the two subgroups was not significantly different $(\mathrm{OR}=1.25 ; 95 \% \mathrm{Cl}, 0.76-2.06 ; \mathrm{p}=0.08)$. Moreover, moderate heterogeneity could be detected between the initially resectable metastatic tumor and the initially unresectable metastatic tumor subgroups, suggesting that different included subjects might result in distinct effects on the $\mathrm{R} 0$ resection rates. Comparatively, the sample size in the initially resectable metastatic tumor subgroup was too small. On the other hand, our pooled analysis also showed that cetuximab combined with different chemotherapy regimens might render different PFS. Besides, it could be found when combining the seven RCTs that PFS in systemic chemotherapy combined with cetuximab group was slightly increased $(\mathrm{HR}=0.88 ; \mathrm{p}<0.05 .95 \% \mathrm{Cl}, 0.77-1.00 ; \mathrm{p}=0.05)$; however, no statistically significant difference was observed in OS between the two groups $(\mathrm{HR}=0.98 .95 \% \mathrm{Cl}, 0.86-1.11$; $p=0.04)$. Therefore, these results indicated that systemic chemotherapy combined with cetuximab exert no marked effect on improving PFS, OS, and surgical resection rate in KRAS wild-type tumors.

In this meta-analysis, the potential heterogeneity was minimised through limiting the study design, sample characteristics, and chemotherapy strategies. Nevertheless, there was certain heterogeneity in the initially unresectable metastatic tumor group $\left(I^{2}=58 \%\right)$, but the results remained unchanged after adjusting for heterogeneity.

Liver is the primary metastatic site of CRC, and the 5-year survival rate after surgical resection is $24-58 \%$, with the average of $40 \%$. At present, surgical resection remains the preferred choice due to the obvious survival benefit. Notably, some patients with initially unresectable lesions may convert to resectable ones following the induction of chemotherapy, and the conversion rate depends on some factors, including the subjective assessment of resectability by the surgeon. Specifically, the conversion rate of patients with truly unresectable lesions is about 5-15\%. FOFIRI with or without cetuximab or panimab, or FOLFOX with or without panimab, has been recommended by the latest NCCN guidelines for neoadjuvant therapy in patients with initially resectable wild-type KRAS liver metastases. Nonetheless, it remains controversial whether the combination of cetuximab with oxaliplatinum-based chemotherapy in patients with CRCLM is reasonable. For patients with initially unresectable hepatic metastatic tumor, the scheme with high ORR is usually chosen due to the close correlation of remission rate with surgical resection rate. ${ }^{20,21}$ It has been reported that the FOLFOXFIRI protocol can achieve a high successful resection rate in patients of CRC with initially unresectable liver metastases, but it has not been confirmed about whether the addition of cetuximab to chemotherapy can increase the resection rate of potentially resectable patients and improve their outcomes. ${ }^{22,23}$ MRC COIN trial is the largest study, in which the EGFR-targeted monoclonal antibodies are added to the first-line chemotherapy for advanced CRC patients. However, patients in this trial cohort are at the advanced stage, and a considerable proportion of them are associated with multi-site metastatic diseases.

In the course of treatment, many patients opt to reduced dose significantly, due to adverse reactions; as a result, the full therapeutic benefit may not be realised for the study arm. Yet, it remains a source of controversy about the effect of chemotherapy combined with cetuximab on the prognosis of potentially resectable $\mathrm{mCRC}$. Sufficient data were lacking in the current meta-analysis, and our results had preliminarily shown that the surgical resection rate and $O S$ in patients receiving systemic chemotherapy combined with cetuximab were not increased in relation to those patients receiving chemotherapy alone; but PFS could be improved compared with that in chemotherapy alone group.

Some limitations should be noted in this study. Firstly, only studies published in English were enrolled. Secondly, data extraction was carried out based on the published papers rather than the individual patient data (IPD), while meta-analysis based on IPD would be more reliable than on the extracted data. Thirdly, more RCTs were lacking in this meta-analysis.

\section{CONCLUSION}

This meta-analysis evaluate the clinical efficacy of adding EGFR-targeted monoclonal antibodies to the chemotherapy regimen for patients of KRAS wild-type tumors with poten- 
tially resectable CRCLM. Our results have preliminarily shown that, compared. with patients receiving chemotherapy alone, the surgical resection rate and PFS in those undergoing systemic chemotherapy combined with cetuximab are not increased; however, OS can be improved in relation to that in patients receiving chemotherapy alone. Noteworthily, the tradeoffs between the high cost and potentially increased toxicity of cetuximab added to the chemotherapy regimen, should be further validated in future clinical practice.

\section{CONFLICT OF INTEREST:}

The authors declared no conflict of interest.

\section{AUTHORS' CONTRIBUTION:}

YK: Designed the study.

YK, LH: Wrote the manuscript.

YK, LH, XX: Analysed the data, interpreted the result and gave final approval on the revised version to be published.

\section{REFERENCES}

1. Kopetz S, Chang GJ, Overman MJ, Eng C, Sargent DJ, Larson DW, et al. Improved survival in metastatic colorectal cancer is associated with adoption of hepatic resection and improved chemotherapy. J Clini Oncol 2009; 27(22): 3677-3683. doi: 10.1200/JCO.2008.20.5278.

2. Adam R, Delvart V, Pascal G, Valeanu A, Castaing D, Azoulay $A$, et al. Rescue surgery for unresectable colorectal liver metastases downstaged by chemotherapy: A model to predict longterm survival. Ann Surg 2004; 240(4):644-57; discussion 657-648. doi: 10.1097/ 01.sla.000014 1198.92114.f6.

3. Kanas GP, Taylor A, Primrose JN, Langeberg WJ, Kelsh MA, Mowat FS, et al. Survival after liver resection in metastatic colorectal cancer: Review and meta-analysis of prognostic factors. Clinical Epidemiology 2012; 4:283-301. doi: 10.2147/ CLEP.S34285.

4. Alberts SR, Horvath WL, Sternfeld WC, Goldberg RM, Mahoney MR, Dakhil SR, et al. Oxaliplatin, fluorouracil, and leucovorin for patients with unresectable liver-only metastases from colorectal cancer: A north central cancer treatment group phase ii study. J Clin Oncol 2005; 23(36):9243-9. doi: $10.1200 /$ JCO.2005.07.740.

5. Giuliani J, Bonetti A. The pharmacological costs of complete liver resections in unselected advanced colorectal cancer patients: Focus on targeted agents. A review of randomized clinical trials. J Gastrointest Cancer 2016; 47(4):341-50. doi: 10.1007/s12029-016-9862-0.

6. Saltz LB, Meropol NJ, Loehrer PJ, Needle MN, Kopit J, Mayer RJ. Phase II Trial of cetuximab in patients with refractory colorectal cancer that expresses the epidermal growth factor receptor. J Clin Oncol 2004; 22(7):1201-8. doi: 10.1200/JC0.2004.10.182.

7. Cunningham DHY, Siena S, Khayat D, Bleiberg H, Santoro A, Bets $D$, et al. Cetuximab monotherapy and cetuximab plus irino- tecan in irinotecan-refractory metastatic colorectal cancer. N Engl J Med 2004; 351(4):337-45. doi: 10.1056/NEJMoa033025.

8. Jonker DJ, Karapetis CS, Zalcberg JR, Dongsheng TU, Heather JA, Berry SR, et al. Cetuximab for the treatment of. colorectal cancer. N Engl J Med 2007; 357:2040-8.

9. Bokemeyer C, Bondarenko I, Makhson A. Fluorouracil, leucovorin, and oxaliplatin with and without cetuximab in the first-line treatment of metastatic colorectal cancer. J Clin Oncol 2009; 27(5):663-71.

10. Van Cutsem E, Kohne $\mathrm{CH}$, Hitre E, Zaluski J, Rong Chang C, Makhson A, et al. Cetuximab and chemotherapy as an initial treatment of metastatic colorectal cancer. N Engl J Med 2009; 360:1408-1417. doi: 10.1056/NEJMoa0805019.

11. Ye LC, Liu TS, Ren L, Wei Y, Zhu DX, Zai SY, Ye, et al. Randomised controlled trial of cetuximab plus chemotherapy for patients withkraswild-type unresectable colorectal liverlimited metastases. J Clin Oncol 2013; 31(16):1931-8. doi: 10.1200/JCO.2012.44.8308.

12. Primrose J, Falk S, Finch Jones M, Valle J, O'Reilly D, Siriwardena $A$, et al. Systemic chemotherapy with or without cetuximab in patients with resectable colorectal liver metastasis: The new epoc randomised controlled trial. Lancet Oncol 2014; 15(6):601-11. doi: 10.1016/S1470- 2045(14)70105-6.

13. Moher DLA, Tetzlaff J. The PG. preferred reporting items for systematic reviews and meta-analyses: The prisma statement. PLOS Medicine 2009; 6(7):e1000097.

14. Maughan TS, Adams RA, Smith CG, Meade AM, Seymour MT, Wilson $\mathrm{RH}$, et al. Addition of cetuximab to oxaliplatin-based first-line combination chemotherapy for treatment of advanced colorectal cancer: Results of the randomised phase $3 \mathrm{MRC}$ COIN trial. Lancet 2011; 377(9783): 2103-2114. doi: 10.1016/S0140-6736(11)60613-2.

15. Tveit Kjell Magne, Guren T, Glimelius B, Pfeiffer P, Sorbye H. Phase III trial of cetuximab with continuous or intermittent fluorouracil, leucovorin, and oxaliplatin (nordic flox) versus flox alone in first-line treatment of metastatic colorectal cancer: The nordic-VII Study. J Clin Oncol 2012; 30(15): 1755-62.

16. Pugh SA, Bowers M, Ball A, Falk S, Jones MF, Valle JW, et al. Patterns of progression, treatment of progressive disease and post-progression survival in the New EPOC study. British J Cancer 2016; 115(4):420-4. doi: 10.1038/bjc. 2016.208.

17. Jones CTM, McWilliams $B$. The role of cetuximab as first-line treatment of colorectal liver metastases. Hpb 2013; 15(1):11-17. doi: 10.1111/j.1477-2574.2012.00591.x.

18. Folprecht G, Gruenberger T, Bechstein WO, Rudolf Raab H, Lordick F, Hartmann JT, et al. Tumour response and secondary resectability of colorectal liver metastases following neoadjuvant chemotherapy with cetuximab: The CELIM randomised phase 2 trial. Lancet Oncol 2010; 11(1):38- 47. doi: 10.1016/S1470-2045(09)70330-4.

19. Garufi C, Torsello A, Tumolo S. Cetuximab plus chrono-modulated irinotecan, 5-fluorouracil, leucovorin and oxaliplatin as neoadjuvant chemotherapy in colorectal liver metastases: POCHER trial. Br J Cancer 2010; 103(10): 1542-7. doi: 10.1038/sj.bjc.6605940.

20. Folprecht G, Grothey A, Alberts S, Raab HR, Köhne CH. Neoadjuvant treatment of unresectable colorectal liver metastases: Correlation between tumour response and resection rates. Annal Oncol 2005; 16(8):1311-9. doi: 10.1093/annonc/mdi246

21. Wagman LD. Importance of response to neoadjuvant therapy in patients with liver-limited mcrc when the intent is resection and/or ablation. Clin Colorectal Cancer 2013; 12(4):223-32. doi.org/10.1016/j.clcc.2013.06.006.

22. Blazer DG, Kishi Y, Maru DM, Kopetz S, Chun S, Overman MJ, et al. Pathologic response to preoperative chemo-therapy: A new 
outcome end point after resection of hepatic colorectal metastases. J Clin Oncol 2008; 26(33):5344-51. doi: 10.1200/JC0.2008.17.5299.
23. Adam RAT, Lévi F. Hepatic resection after rescue cetuximab treatment for colorectal liver metastases previously refractory to conventional systemic therapy. J Clin Oncol 2007; 25(29):4593-4602.

$\bullet \bullet \bullet \bullet \bullet \bullet \bullet \bullet \bullet$ 